\title{
Établissement d'une cryobanque d'embryons pour la conservation ex situ de la diversité génétique chez le lapin : aspects pratiques
}

\author{
Thierry Joly ${ }^{\mathrm{a}, \mathrm{b} *}$, Hubert de Rochambeau ${ }^{\mathrm{c}}$, Jean-Paul Renard ${ }^{\mathrm{a}}$ \\ ${ }^{a}$ Inra, biologie du développement, 78352 Jouy-en-Josas, France \\ ${ }^{b}$ Isara, 31 place Bellecour, 69288 Lyon cedex 02, France \\ c Inra, Saga, BP 27, 31326 Castanet Tolosan, France
}

\begin{abstract}
Cryobanking of embryos to preserve ex situ rabbit genetic resources : practical aspects. Embryo cryobanks are a valuable tool for maintaining the genetic variability in domestic animal populations. Such a bank was built for the rabbit (Oryctolagus cuniculus), a multiple purpose species, which is bred for its meat, wool and fur as well as biomedical research. Several populations were selected to sample the whole genetic diversity. This paper aims firstly at evaluating the number of young rabbits born from one batch of frozen embryos. Forty newborn rabbits were obtained from 100 embryos. This result varies from one to two between populations. However, the number of bucks and does necessary to produce one batch of embryos varies from one to three between populations. Later, we will have to address the problem of the size of the population that will produce the embryos and whether to store one gene or to preserve the genetic variability of one population. (C) Inra/Elsevier, Paris
\end{abstract}

cryobank / embryo / rabbit / sampling / genetic resources

Résumé - Les cryobanques d'embryons peuvent apporter une aide précieuse au maintien de la diversité génétique des populations d'animaux domestiques. Nous avons mis en place une telle banque chez le lapin (Oryctolagus cuniculus), espèce d'intérêt zootechnique, mais aussi modèle animal pour la recherche biomédicale. Après avoir choisi plusieurs populations de lapins pour représenter au mieux la diversité génétique chez cette espèce, nous avons d'abord déterminé quel était le nombre de lapereaux obtenus à partir d'un nombre donné d'embryons congelés. Nos résultats montrent qu'en moyenne 40 lapereaux sont produits après décongélation de 100 embryons. Ce taux varie du simple au double d'une population à l'autre. Ensuite, selon la population considérée, le nombre d'animaux nécessaire pour produire ces embryons

\footnotetext{
* Correspondance et tirés à part
} 
varie du simple au triple. À partir de ces données, il est possible de définir pour chaque population de lapin retenue les caractéristiques de l'échantillon d'animaux à utiliser comme donneurs d'embryons. (c) Inra/Elsevier, Paris

cryobanque / embryon / lapin / échantillonnage / ressources génétiques

\section{INTRODUCTION}

Les cryobanques d'embryons peuvent apporter une aide précieuse au maintien de la diversité génétique des populations d'animaux domestiques. Nous avons mis en place une telle banque chez le lapin (Oryctolagus cuniculus), espèce d'intérêt zootechnique, mais aussi modèle animal pour la recherche biomédicale.

Le but prioritaire d'une cryobanque est de conserver en l'état initial les potentialités évolutives d'une population. Ainsi, la cryobanque doit constituer une structure qui rassemble et conserve un stock d'embryons suffisant pour représenter la population initiale à l'état congelé, pendant une période qui peut s'étendre de quelques jours à plusieurs dizaines d'années. En effet, il a été montré qu'à une température de $-196^{\circ} \mathrm{C}$, les embryons de souris pouvaient être conservés pendant plus de 20 ans sans modifier leur aptitude à donner des descendants normalement fertiles (Glenister et al., 1990; Dulioust, 1995). L'autre but, tout aussi important, est lié à l'activité d'échange, consécutive à une utilisation des ressources dissociée dans le temps et dans l'espace. La cryobanque doit permettre d'échanger du matériel biologique congelé avec un maximum de garantie sur le plan sanitaire. D'une façon générale, la mise en place d'une cryobanque inclut la mise en œuvre de l'ensemble des moyens nécessaires au maintien des potentialités évolutives des populations. Au sens large, cette structure doit être considérée comme un outil de la gestion animale pour le maintien de la diversité génétique des animaux domestiques. La cryobanque est complémentaire aux actions de conservation in situ et représente beaucoup plus qu'un stock d'embryons, simple support pour des opérations de muséologie visant uniquement à figer les produits du passé. Le déroulement d'une telle action entraîne la prise en compte des aspects génétiques, physiologiques, sanitaires, socio-économiques et juridiques.

Depuis plusieurs années, de nombreux acteurs ont pris en compte la perte de la diversité génétique animale et ont œuvré pour la sauvegarder. Cependant, la situation des ressources génétiques animales diffère considérablement suivant les espèces animales et les acteurs impliqués. En effet, des travaux sur les espèces d'animaux sauvages ont été mis en œuvre, essentiellement à la demande des parcs zoologiques et concernent principalement les espèces exotiques africaines (Wildt, 1992) et quelques espèces européennes (Loskutoff et al., 1993). De même, la conservation des animaux modèles de recherche font l'objet d'initiatives individuelles dans le domaine de la recherche biologique et médicale. Ces espèces (murins, oncins,...) sont maintenues en petites colonies vivantes pour la durée des expériences avant d'être éventuellement conservées à l'état de cellules congelées dans des cryobanques privées (Mobraaten, 1986; Gershon, 1993). Dans ces deux situations, les ressources génétiques animales font l'objet de plans de conservation bien structurés, sans problème majeur de financement des programmes. La situation est différente concernant les nombreuses populations traditionnelles et les races identifiées, pour la plupart soumises aux pressions des marchés économiques et financiers. Depuis sa création, la FAO a 
œuvré entre autres dans le domaine de la conservation, l'évaluation et l'utilisation des ressources génétiques animales (Hodges, 1990). Dernièrement, la FAO a établi une infrastructure internationale qui regroupe sept banques régionales de gènes animaux répartis sur l'ensemble des continents. Ces banques ont pour mission de stocker, en priorité, la semence et les embryons des animaux issus de populations ou de races menacées de disparition.

Notre travail a pour but de fournir une base scientifique et technique pour établir une cryobanque. Le choix du lapin s'explique pour des raisons économiques (coûts modérés des animaux,...), des raisons pratiques (technique de congélation d'embryons maîtrisée...) et des raisons génétiques. Il existe une grande diversité de populations dans cette espèce : animaux sauvages, domestiques, de laboratoire et de compagnie. Les données physiologiques obtenues sur plusieurs populations de lapins permettent de répondre à deux questions importantes pour définir la taille de l'échantillon.

- Quel est le nombre d'animaux reproducteurs nécessaire pour produire un nombre donné d'embryons congelés? Nous avons utilisé deux modes de production d'embryons, la superovulation et la non-stimulation, pour disposer de références sur l'apport de ces méthodes à la constitution d'une cryobanque.

- Quel est le nombre de lapereaux que l'on peut obtenir à partir d'un nombre donné d'embryons congelés?

\section{MATÉRIEL}

Dans le cadre de notre action, plusieurs populations ont été choisies afin de représenter au mieux la diversité chez cette espèce et d'illustrer les différentes questions rencontrées lors de la mise en place des actions de cryoconservation des ressources génétiques animales. Les caractéristiques de ces populations et l'intérêt de leur cryoconservation sont présentés dans le tableau $I$. Le recours aux cryobanques pour conserver ex situ ces populations s'explique principalement par des motifs de sécurité, face à une perte accidentelle d'animaux précieux, des motifs économiques, la cryoconservation des embryons étant moins coûteuse que la conservation d'animaux vivants, et des motifs socioculturels.

Au total, près de 610 animaux appartenant à sept populations différentes ont été échantillonnés pour notre travail expérimental. Ces animaux âgés de 4,5 mois à 5 ans sont placés dans des conditions d'environnement et des systèmes d'élevage différents. Les lapins de race Brun Marron de Lorraine et les lapins sauteurs d'Alfort sont élevés dans des élevages traditionnels, en clapier sur litière paillée. Les lignées commerciales (A1077, Deutsch Petit Russe, néozélandaise) et les animaux modèle de recherche (lignées allotypées de Bâle, A1029) sont élevés en batterie dans des unités hors sol aux conditions de température et d'hygrométrie contrôlées.

\section{MÉTHODES}

\subsection{Production des embryons}

La production d'embryons par superovulation consiste à administrer à chaque femelle une dose totale de $2 \mathrm{mg}$ de pFSH (Stimufol, Rhône Mérieux, 


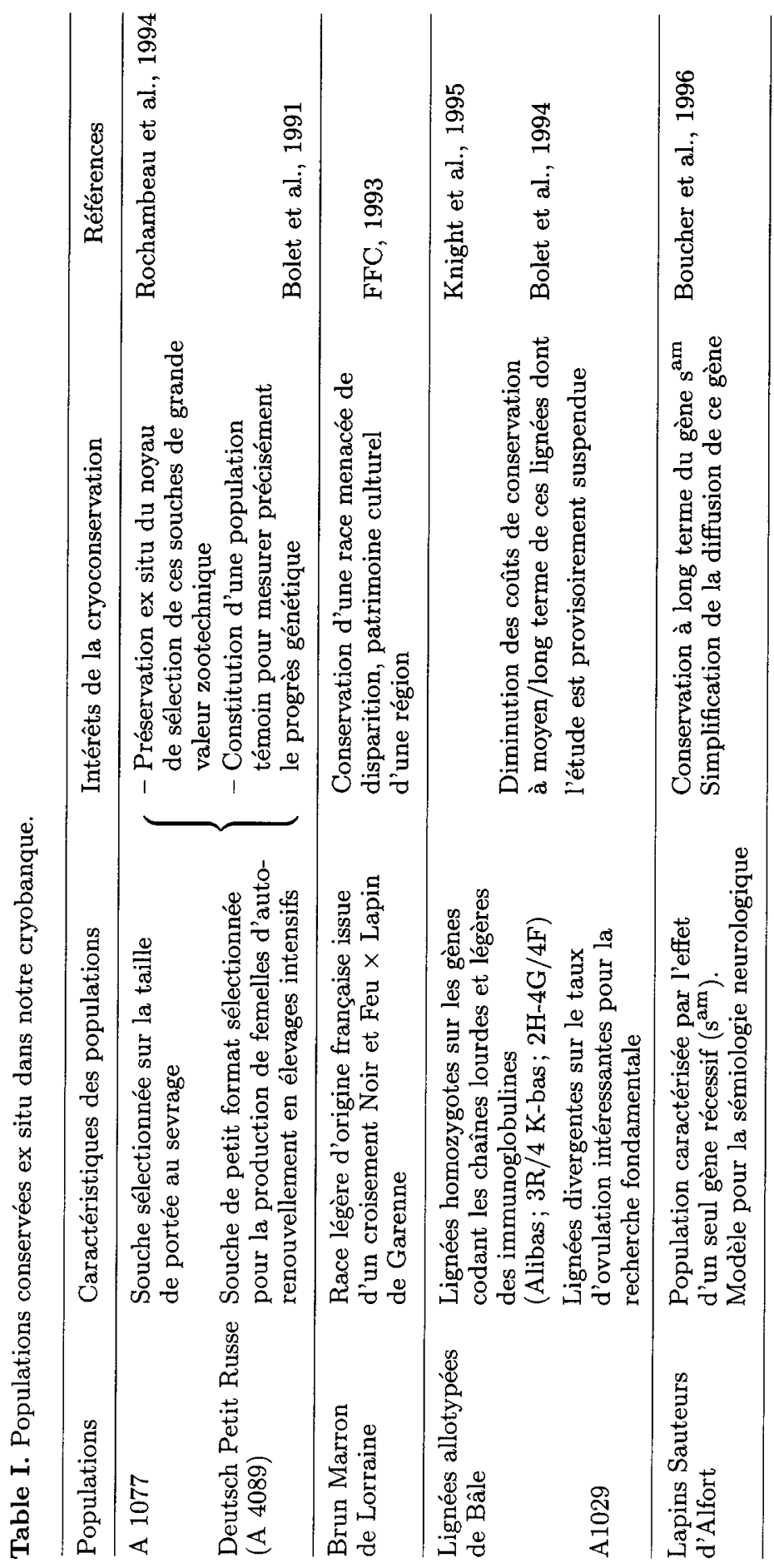


France) en cinq injections à $12 \mathrm{~h}$ d'intervalle. Les femelles sont fécondées soit par insémination artificielle, soit par accouplement et l'ovulation est renforcée ou induite par une injection d'un analogue de $\mathrm{GnRH}(0,3 \mathrm{~mL}$ de buséréline; Réceptal, Distrivet, France). L'un des principaux avantages du traitement de superovulation est de permettre la synchronisation d'un groupe de femelles pour une même expérience de collecte d'embryons, et ce, quel que soit leur état de réceptivité. En revanche, la production d'embryons sans superovulation concerne uniquement les lapines sexuellement réceptives. Immédiatement après la fécondation des femelles, une dose de $0,2 \mathrm{~mL}$ de buséréline (Réceptal) est injectée.

Les embryons sont collectés au stade morula compactée, 65 à $72 \mathrm{~h}$ postcoïtum après abattage des femelles et perfusion du tractus génital à l'aide d'une solution saline tamponnée au phosphate (PBS).

\subsection{Congélation des embryons}

La méthode de congélation retenue implique l'emploi d'un congélateur programmable (Cryocell 1200 ; IMV, L'Aigle, France) et son maniement simple, en fait un appareil bien adapté pour intervenir directement dans les élevages (Joly et al., 1994). La méthode employée comprend trois étapes : la première consiste à plonger les embryons dans trois bains successifs de PBS contenant respectivement $0,5 \mathrm{M}, 1,0 \mathrm{M}$ et $1,5 \mathrm{M}$ de DMSO pendant $5 \mathrm{~min}$ à température ambiante, avant de les monter dans une paillette plastique identifiée $(0,25 \mathrm{~mL}$, $\mathrm{IMV})$; dans un deuxième temps, les paillettes sont équilibrées à $-7^{\circ} \mathrm{C}$ pendant 5 min avant l'induction de la cristallisation; la troisième étape consiste à abaisser lentement la température à une vitesse de $0,5^{\circ} \mathrm{C}$ par min jusqu'à $-30^{\circ} \mathrm{C}$ puis immerger les paillettes directement dans l'azote liquide à $-196^{\circ} \mathrm{C}$.

\subsection{Décongélation et transfert des embryons}

Après décongélation dans un bain-marie à $20^{\circ} \mathrm{C}$ pendant $30 \mathrm{~s}$, les embryons sont plongés successivement pendant 5 min dans deux bains de PBS contenant respectivement 1,0 M et 0,5 M de DMSO puis ensuite rincés dans trois bains de PBS. Les embryons dégénérés sont éliminés, alors que ceux considérés viables après observation de leur morphologie (sans dommages cellulaires apparents) sont transférés dans des receveuses synchronisées par une injection de $0,2 \mathrm{~mL}$ de buséréline (Receptal). Les embryons (huit à douze embryons par receveuse) sont déposés par voie chirurgicale directement dans les cornes utérines, $65 \mathrm{~h}$ après l'induction de l'ovulation (Techakumphu et al., 1987). La viabilité des embryons est évaluée par leur capacité à devenir des lapereaux vivants à la mise bas. Les femelles utilisées comme receveuse appartiennent à des lignées commerciales avec des performances de reproduction similaires (A1077, A1029, NZ) et sont placées dans des conditions d'élevage hors sol standard.

\section{RÉSULTATS}

\subsection{Production des embryons congelés (tableau II)}

Dans l'ensemble, 462 femelles ont été traitées (320 superovulées et 142 non superovulées) et ont permis de congeler au total 4672 embryons. Suivant les 
Table II. Efficacité des méthodes de production d'embryons chez les femelles traitées.

\begin{tabular}{lrrrrr}
\hline $\begin{array}{l}\text { Type de } \\
\text { population }\end{array}$ & \multicolumn{2}{c}{$\begin{array}{c}\text { Nombre de femelles } \\
\text { Traitées }\end{array}$} & \multicolumn{3}{c}{$\begin{array}{c}\text { Embryons congelés } \\
\text { Donneuses (\%) }\end{array}$} \\
& & & & $\begin{array}{r}\text { Total donneuse } \\
\text { moy es }\end{array}$ \\
\hline Traitement de superovulation & & & & & \\
Brun marron de Lorraine & 34 & $22(65 \%)$ & 300 & $13,6 \pm 1,9$ & {$[1-32]$} \\
A 1077 & 72 & $49(68 \%)$ & 1250 & $25,5 \pm 2,2$ & {$[2-75]$} \\
A 1029 & 134 & $103(77 \%)$ & 1589 & $15,4 \pm 0,9$ & {$[1-44]$} \\
Lignées allotypées de Bâle & 50 & $27(54 \%)$ & 295 & $10,9 \pm 1,5$ & {$[1-34]$} \\
Lignées histocompatibles US & 20 & $10(50 \%)$ & 171 & $17,1 \pm 4,1$ & {$[4-50]$} \\
Lapins Sauteur d'Alfort' & 10 & $9(90 \%)$ & 274 & $30,4 \pm 5,1$ & {$[6-50]$} \\
Total & 320 & $210(70 \%)$ & 3879 & 18,5 & \\
Sans superovulation & & & & & \\
A 1077 & 53 & $43(81 \%)$ & 352 & $8,2 \pm 0,6$ & {$[1-17]$} \\
A 1029 & 39 & $26(67 \%)$ & 207 & $8,0 \pm 0,6$ & {$[3-14]$} \\
Deutsch Petit Russe & 50 & $31(62 \%)$ & 234 & $7,5 \pm 0,5$ & {$[1-13]$} \\
Total & 142 & $100(70 \%)$ & 793 & 7,9 & \\
\hline
\end{tabular}

$\left(^{*}\right)=$ moyenne \pm erreur standard [minimum maximum].

populations, ces opérations se sont déroulées dans des laboratoires, dans des stations expérimentales ou directement dans des fermes d'élevage. L'efficacité des méthodes de production d'embryons est définie à partir du pourcentage de femelles donneuses (une femelle donneuse est une femelle traitée ayant des embryons congelés) et du nombre d'embryons congelés par donneuse. Quelle que soit la méthode de production des embryons (avec ou sans traitement de superovulation), $70 \%$ des femelles traitées ont des embryons congelés, mais un écart très important variant de $50 \%$ à $90 \%$ est observé suivant les populations. Le nombre d'embryons congelés par donneuse est en moyenne de 18,5 pour les femelles superovulées et de 7,9 pour les femelles non superovulées. Ces résultats sont variables suivant les populations $([10,9 \sim 30,4]$ pour les femelles superovulées; [7,5 8,2] pour les femelles non superovulées) mais aussi suivant les individus traités ([1 75] pour les femelles superovulées; [1 17] pour les femelles non superovulées). Quel que soit le traitement de production d'embryons, ces résultats peuvent s'expliquer en partie par les variations du taux de collecte [écart : $60 \% \sim 93 \%$ ] et du pourcentage d'embryons congelés [56\% 90\%]. Néanmoins, malgré une plus grande variabilité, les femelles superovulées fournissent en moyenne deux fois plus d'embryons congelés par donneuse que les femelles non superovulées.

La distribution du nombre d'embryons congelés et de paillettes par femelle est présentée dans la figure 1. Pour les deux traitements, près de $30 \%$ des femelles échantillonnées ne donnent aucun embryon congelé pour les raisons suivantes : absence d'ovulation $(C J=0)$, ovocytes non fécondés, embryons dégénérés, tractus génital infecté, problèmes au moment de la collecte des embryons,... La distribution montre que $56 \%$ des femelles superovulées et $42 \%$ des femelles non superovulées ont fourni au moins une paillette d'embryons 


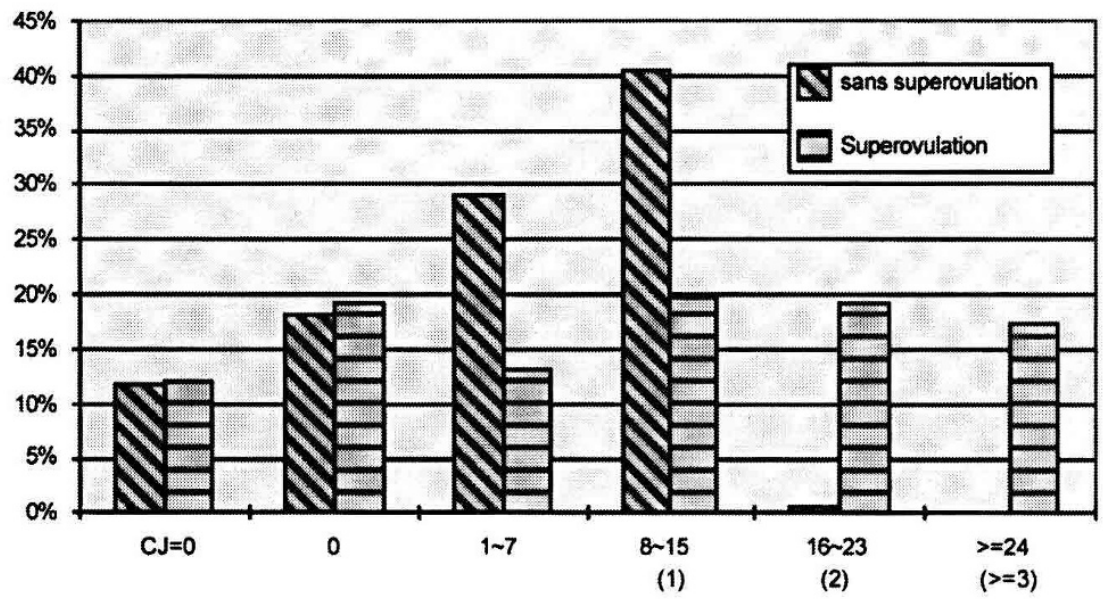

Nombre d'embryons congelés (paillettes) par femelle traitée

Figure 1. Distribution des embryons congelés et des paillettes par femelle traitée (superovulation ou sans superovulation). $C J=0$ signifie qu'il n'y avait aucun corps jaune apparent sur les ovaires des femelles traitées et donc aucun embryon récupéré.

congelés (soit huit embryons) et que plus du tiers des femelles superovulées en ont fourni au moins deux (soit seize embryons).

Ainsi, la superovulation est la méthode la plus intéressante et efficace pour augmenter la production d'embryons congelés par femelle lors d'opérations de cryoconservation de populations de lapins.

\subsection{Viabilité des embryons après décongélation et transfert (tableau III)}

Sur un total de 1357 embryons décongelés, 1306 embryons (96\%) ont été transférés sur 144 receveuses élevées dans deux stations expérimentales distinctes. Dans l'ensemble, $80 \%$ (115/144) des receveuses ayant reçu des embryons décongelés donne naissance à des lapereaux vivants. Le taux de mise bas varie de $59 \%$ à $92 \%$ suivant le génotype des receveuses. Ce taux tient compte des femelles palpées vides à J12 (12\%) mais aussi des accidents divers (chocs opératoires, avortements, mortalité en élevage...). L'efficacité globale du transfert embryonnaire est définie par le taux de développement à terme des embryons congelés (nombre de lapereaux nés vivants/embryons décongelés) : 556 lapereaux sont nés vivants à partir de 1357 embryons congelés, soit $41 \%$. Ce rendement varie du simple au double [23,5\% 51,3\%] suivant le génotype des embryons et des receveuses et semble être étroitement lié au taux de mise bas des receveuses. Dans l'ensemble, pour 100 embryons stockés dans la cryobanque, nous pouvons prévoir d'obtenir 40 lapereaux vivants après transfert dans des femelles receveuses. 


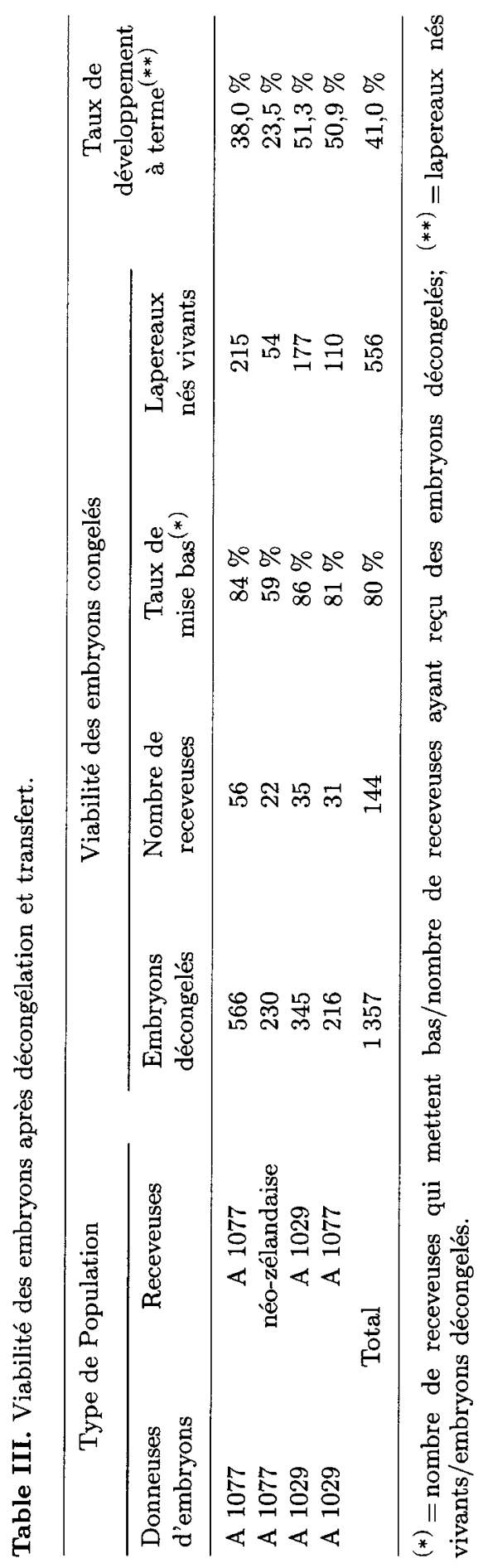


La distribution des donneuses d'embryons en fonction du nombre de leurs descendants est présentée dans la figure 2. Au total, 556 lapereaux nés vivants sont les descendants de 83 donneuses (femelles ayant donné au moins huit embryons congelés), soit un nombre moyen de descendants par donneuse de 6,7 (556/83). La distribution montre que $84,3 \%$ des donneuses (70/83) ont eu au moins un descendant vivant et $49,4 \%$ (41/83) plus de six lapereaux. En définitive, ces différents éléments nous permettent de déduire qu'après congélation d'au moins huit embryons, près de la moitié des accouplements donnent six descendants viables ou plus. Compte tenu du sex-ratio, de la mortalité entre la naissance et la puberté, et des critères de sélection des jeunes reproducteurs en fonction de leur standard ou de leur ardeur sexuelle, cette valeur de six lapereaux nés vivants est compatible avec l'obtention d'un couple de reproducteurs adultes.

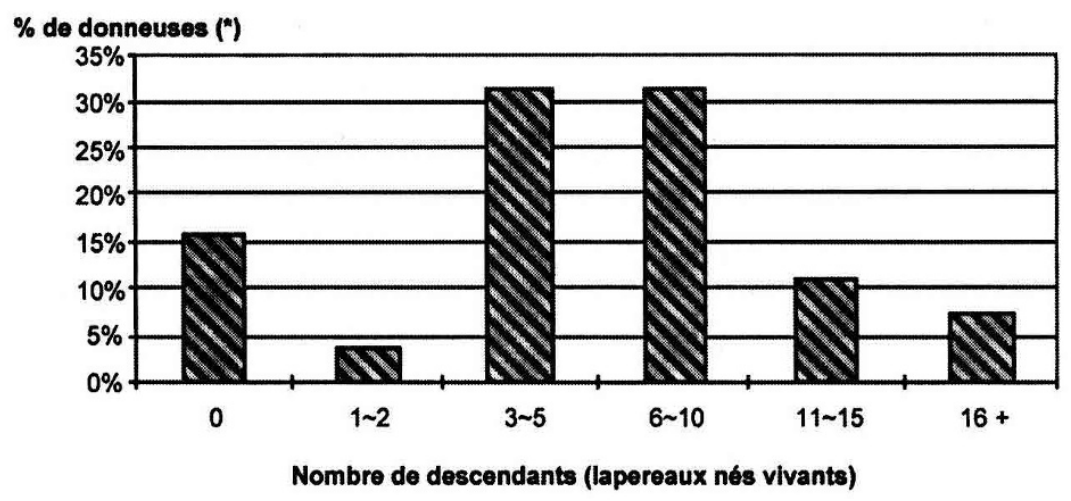

Figure 2. Distribution des donneuses d'embryons en fonction du nombre de leurs descendants (lapereaux nés vivants); ${ }^{*}$ donneuses qui ont donné au moins huit embryons congelés et transplantés dans des receveuses.

\section{DISCUSSION}

Nos résultats montrent que la variabilité observée entre les populations est très élevée : du simple au triple pour les critères de production des embryons (pourcentage de donneuses et nombre d'embryons par donneuse) et du simple au double pour les critères de reconstitution des embryons (taux de mise bas des receveuses et taux de développement à terme des embryons décongelés). Cette variabilité peut s'expliquer par la diversité des populations conservées qui sont toutes de constitutions génétiques très différentes. En effet, certaines populations sont issues d'un long schéma de sélection rigoureux sur des performances de reproduction, d'autres populations maintenues en petit effectif sont consanguines et ont des difficultés de reproduction en conditions naturelles... Une variabilité encore plus grande que celle que nous avons observée chez le lapin a été montrée lors de la mise en place d'une cryobanque d'embryons de souris à l'Institut national de la santé américaine (National Institut of Health, Animal Genetic Resource) : le pourcentage de souris donneuses d'embryons varie dans un rapport de 1 à 10 (moyenne $=30 \%$; écart $=6 \%$ à $70 \%$ ) 
et le nombre moyen d'embryons congelés par donneuse varie dans un rapport de 1 à 4 (moyenne $=12$; écart $=4,7$ à 20,9), (Schmidt et al., 1996). Cependant, cette variabilité n'est pas toujours observée chez d'autres espèces. Lors d'opérations de cryoconservation de quatre populations ovines, les résultats de production d'embryons (nombre d'embryons congelables/collecte) et de reconstitution (taux de développement embryonnaire) n'ont montré aucune différence significative entre les différentes populations. Toutefois, il est à signaler que ces quatre lignées sont toutes issues de schéma de sélection sur l'aptitude à la reproduction et les performances de croissance (Sakul et al., 1993).

Dans toutes opérations de cryoconservation, deux effets sont à prendre en considération : l'effet donneur et l'effet receveuse. L'effet donneur, relatif à l'étape de constitution du stock d'embryons congelés dans la cryobanque, peut se définir par une forte variabilité des critères de production d'embryons suivant le génotype des donneuses. Cette variabilité ne peut pas être maîtrisée a priori car elle est directement liée à la réponse individuelle de chacun des individus traités et à la constitution génétique particulière de chaque population à conserver. Seule, l'organisation des opérations de récolte permet de pallier partiellement cet inconvénient. L'effet receveuse intervient au moment de l'utilisation du stock d'embryons de la cryobanque. Il peut se définir par l'influence prédominante du génotype de la femelle receveuse sur la production de lapereaux vivants (Vicente et al., 1993). Afin de maîtriser ou atténuer partiellement cette variabilité, nous proposons d'utiliser une population génétiquement bien caractérisée, la souche A1077 comme receveuse universelle.

La connaissance de la variabilité de ces résultats permet de définir le nombre d'embryons à congeler et l'effectif minimal de mâles utilisés et de femelles collectées dans la perspectives de recouvrir après décongélation des embryons, une proportion déterminée à l'avance de la variabilité génétique présente dans la population. Les calculs que nous avons effectués (Joly, 1997) indiquent que, d'une population à l'autre, les effectifs minimaux sont de l'ordre de 120 embryons si l'objectif est la conservation d'un allèle particulier à un locus donné et de 330 embryons issus de 15 mâles et 30 femelles si l'objectif est la conservation de combinaisons génétiques impliquant plusieurs locus.

\section{CONCLUSIONS, PERSPECTIVES}

Nos techniques de production et de congélation d'embryons peuvent être utilisées en routine pour conserver les ressources génétiques chez le lapin bien que les variabilités individuelles et entre populations observées sur nos résultats de production d'embryons ne soient pas maitrisables.

La cryobanque d'embryons est aujourd'hui une réalité chez le lapin qui contient plus de 5000 embryons provenant de plus de 20 populations et la gestion informatisée d'une telle collection est actuellement en cours. Dans le cadre du programme européen de caractérisation et de conservation des ressources génétiques chez le lapin (RESGEN1-CT95-0060) nous sommes en train de définir avec nos collègues des autres pays une méthode commune d'identification ainsi qu'une stratégie de gestion. Il est maintenant urgent de préciser le cadre juridique de façon à pouvoir accueillir dès 1998 des embryons provenant de différents pays d'Europe. 


\section{RÉFÉRENCES}

Bolet G., Rochambeau H. de, Coudert P., Caractéristiques génétiques des souches de lapins de l'Inra. 11 e Journées d'Études IFFA Credo le 3-4 octobre 1991, Marcy l'Étoile, France, Fondation M. Mérieux, Lyon, 35-52, (1991).

Bolet G., Santacreu M.A., Argente M.J., Climent A., Blasco A., Divergent selection for uterine efficiency in unilaterally ovariectomized rabbits. I. Phenotypic and genetic parameters. 5th World Congress on Genetic Applied to Livestock Production, Guelph, Canada, 11 août 1994, XIX, 261-264, 1994.

Boucher S., Renard JP., Joly T., The'Alfort Jumper rabbit : historic, description and characterization. 6th World Rabbit Congress, Toulouse 9-12 July 1996, Association Scientifique Française de Cuniculture, Lempdes (France), 2, 255-258, 1996.

Dulioust E., Long-term effects of embryo freezing in mice, Proc. Natl. Acad. Sci. USA 92 (1995) 589-593.

F.F.C., Standard officiel des lapins de races. Fédération Française de Cuniculture, Paris, 253 p., 1993. 684.

Gershon D., NIH to support embryo bank for mice and rabbits, Nature 362 (1993)

Glenister P.H., Whittingham D.G., Wood M.J., Genome cryopreservation : a valuable contribution to mammalian genetic research, Genet. Res. Camb. 56 (1990) 253-258.

Hodges, J., Manual on establishment and operation of animal gene banks. Rome : Food Agriculture Organization of the United Nations, 67 p., (1990).

Joly T., Theau-Clement M., Drouet-Viard F., Rochambeau H. de, Renard JP., Application de la cryoconservation des embryons à la protection des ressources génétiques chez le lapin, Genet. Sel. Evol. 26 (1994) Suppl 1, 267-278.

Joly T., Établissement d'une cryobanque de semence ou d'embryons pour la conservation ex situ de la diversité génétique chez les mammifères domestiques : l'exemple du lapin, thèse, Insa, Lyon, 143 p., 1997.

Knight K.L., Burnett R.C., Nicholas J.M., Organization and polymorphism of rabbit immunoglobulin heavy chain genes, J. Immunol. 134 (1985) 1245-1250.

Loskutoff N.M., Bartels P., Meintjes M., Godke R.A., Schiewe M.C., Assisted reproductive technology in non-domestic ungulates : a model approach to preserving and managing genetic diversity, Theriogenology 43 (1995) 3-12.

Mobraaten L.E., Mouse embryo cryobanking, J. in vitro Fertilization and Embryo Transfer 39 (1986) 401-409.

Rochambeau H. de, Bolet G., Tudella F., Long-term selection : comparison of two rabbit strains. 5th World Congress on Genetics Applied to Livestock Production, Guelph, Canada, 11 Aug. 1994, XIX, 257-260.

Sakul H., Bradford G.E., Bondurant R.H., Anderson G.B., Donahue S.E., Cryopreservation of embryos as a means of germ plasm conservation in sheep, Theriogenology 39 (1993) 401-409.

Schmidt M.P., Lin X., Brown S.S., Wallace G.A., Rall W.F., Hansen C.T., The genotypic variability of banking embryos from mouse models in the NIH animal genetic resource, Theriogenology 45 (1996) 171.

Techakumphu M., Heyman Y., Survival of frozen-thawed rabbit morulae after synchronous or asynchronous transfer, Anim. Reprod. Sci. 12 (1987) 305-312.

Vicente J., Garcia-Ximenez F., Effect of recipient doe genotype on survival rate at birth of frozen rabbit embryos, Reprod. Nutr. Dev. 33 (1993) 229-234.

Wildt D.E., Genetic resource banks for conserving wildlife species : justification, examples and becoming organized on a global basis, Anim. Reprod. Sci. 28 (1992) 247-257. 